\title{
InfoSecRM: Uma Abordagem Ontológica para a Gestão de Riscos de Segurança da Informação
}

\author{
Éder S. Gualberto ${ }^{1}$, Rafael T. de Sousa Jr ${ }^{1}$, Flávio E. G. de Deus ${ }^{1}$, Cláudio G. Duque ${ }^{2}$ \\ ${ }^{1}$ Departamento de engenharia Elétrica - Universidade de Brasília (UnB) \\ Caixa Postal 4.386 - 70.910-900 - Brasília - DF - Brazil \\ ${ }^{2}$ Faculdade de Ciência da Informação (FCI) \\ Universidade de Brasília (UnB) - Brasília - DF - Brazil \\ \{edergual\}@gmail.com, \{rafael,flavioelias,klauss\}@unb.br
}

\begin{abstract}
Information Security Risk Management processes uses information from various sources such as data about assets and their vulnerabilities, system logs, manager decisions. Resources that can help in information handling of this complex framework are real and relevant needs to be considered. In this paper, an ontology is presented as a representation proposal to formalize, share, manipulate and process concepts and related information to the field of information security risk management.
\end{abstract}

Resumo. Processos de Gerenciamento de Riscos de Segurança da informação utilizam informações de fontes variadas como dados sobre ativos e suas vulnerabilidades, logs de sistemas, decisões gerenciais etc. Recursos que possam auxiliar na manipulação de informações deste complexo arcabouço são necessidades reais e relevantes a serem consideradas. Neste artigo, é apresentada uma ontologia como proposta de representação para formalizar, compartilhar, manipular e processar conceitos e informações relacionadas ao domínio de gestão de riscos de segurança da informação.

\section{Introdução}

Promover e gerir a segurança da informação têm sido um grande desafio para as organizações, visto a alta criticidade deste tipo de ativo e dos ativos de suporte relacionados, e as constantes ameaças que os cercam [Sêmola 2003]. Neste sentido, considerando que esta prática vai além de questões inerentemente relacionadas à tecnologia da informação (TI), ações gerenciais que baseiam-se em processos, tecnologias e pessoas são importantíssimas para a efetividade da segurança da informação.

Assim, a questão da gestão da segurança da informação (GSI) em ambientes corporativos, públicos e privados, tem sido bastante discutida, prova disso foram as publicações das normas da família ISO 27000 que são, conforme afirmado em [ISO 2009], o estado da arte internacional nesta área. Estas normas definem um modelo para sistemas de gerenciamento de segurança da informação (SGSI) com vistas a proteger os ativos de informação e permitir à organização continuar realizando sua missão.

No âmbito de um SGSI, um processo de gestão de riscos de segurança da informação (GRSI) caracteriza-se como um dos elementos mais importantes para sua efetividade, visto que o mesmo permite a identificação das necessidades e prioridades de 
segurança da informação da organização com base em análises e avaliações que se guiam pelos critérios e requisitos definidos pela própria organização. Segundo [ABNT 2008], administrar os riscos de segurança da informação aos quais se está sujeito contribui de maneira significativa para o sucesso da organização e de seus negócios.

Ocorre no entanto que a implementação e manutenção de um processo de GRSI opera sobre uma grande quantidade de conceitos. Conceitos estes que manifestam muitos relacionamentos entre si, tornando seu entendimento e aprendizado, por parte dos colaboradores e intervenientes (stakeholders), fator crítico à aquisição e ao compartilhamento de conhecimento relativo à segurança da informação da organização. Além disso a GRSI atua sobre uma grande quantidade de informações e a utilização de mecanismos que permitam a sua manipulação de forma eficiente e eficaz são extremamente relevantes, [AS/NZS 2004].

O conhecimento em segurança vale-se de variadas fontes de informação e estruturar estas informações de modo a permitir o processamento, o compartilhamento e a utilização deste conhecimento é uma tarefa complexa, define [Schumacher 2003]. Assim, paradigmas que promovam a automação deste processo e possibilitem a definição da arquitetura da informação relacionada são representações extremamente últeis às modelagens deste tipo de domínio.

Neste cenário, a utilização de ontologias permite, ao mesmo tempo, a representação das relações semânticas entre os conceitos envolvidos em um processo de GRSI e de GSI, e a criação e estruturação de uma base de conhecimento a respeito da segurança da informação na organização, além de possibilitar a comunicação e interoperabilidade entre agentes de software e agentes humanos sobre uma mesma representação de dados.

Diante o exposto, foi desenvolvida uma ontologia para gestão de riscos de segurança da informação denominada InfoSecRM. Essa caracteriza-se como uma ontologia de domínio, visto que dispõe dos conceitos básicos relacionados ao domínio de GRSI e de GSI. Por meio da utilização dessa, pode-se documentar e operar o processo de GRSI e subsidiar decisões gerenciais relacionadas à GSI.

\section{Segurança da Informação}

A segurança da informação diz respeito à proteção da informação em suas propriedades (confidencialidade ${ }^{1}$, integridade ${ }^{2}$ e disponibilidade ${ }^{3}$ ) e em seus aspectos (autenticidade ${ }^{4}$, legalidade ${ }^{5}$ etc), evitando que as vulnerabilidades dos ativos relacionados sejam exploradas por ameaças e possam trazer consequências para os negócios de uma organização.

Ativos, como definido em [ABNT 2006], refere-se a "qualquer coisa que tenha

\footnotetext{
${ }^{1}$ Propriedade que a informação apresenta, de estar disponível apenas para àqueles que estão autorizados a obtê-la [ABNT 2006].

${ }^{2}$ Propriedade que a informação apresenta, de estar completa e fiel ao estado original [ABNT 2006].

${ }^{3}$ Propriedade que a informação apresenta, de estar disponível e utilizável numa eventual requisição de uma entidade autorizada [ABNT 2006].

${ }^{4}$ Aspecto comprovante de que a informação foi produzida, expedida, modificada ou destruída por uma determinada pessoa física, ou por um determinado sistema, órgão ou entidade [GSI-PR 2008].

${ }^{5}$ Aspecto comprovante do valor legal (onde todos os ativos relacionados estão de acordo com os requisitos de conformidade) que uma informação pode ter em um processo de comunicação [Sêmola 2003].
} 
valor para a organização". Estes ativos possuem fragilidades, denominadas vulnerabilidades [ISO 2009], que podem ser exploradas por ameaças (que segundo [ISO 2009], são as causas potenciais de incidentes de segurança da informação), causando danos e impactos à organização e seus sistemas. Impactos, conforme explicitado em [ISO 2009], "dizem respeito a mudanças adversas nos níveis dos objetivos de negócio alcançados".

A definição de impacto auxilia a diferenciar evento de segurança da informação de incidente de segurança da informação, pois conforme exposto em [ABNT 2005], um evento de segurança da informação caracteriza-se por uma "ocorrência identificada de um sistema, serviço ou rede, que indica uma possível violação da política de segurança da informação ou falha de controles, ou uma situação previamente desconhecida, que possa ser relevante para a segurança da informação", ao passo que incidente de segurança da informação diz respeito apenas aquele(s) evento(s) que tenham grande probabilidade de impactar o negócio e a segurança da informação de uma organização.

Risco, conforme depreende-se de [AS/NZS 2004], refere-se um evento hipotético (com probabilidade de ocorrência não nula), cuja concretização pode afetar de forma positiva ou negativa uma organização. Já o risco de segurança da informação é mais específico, consoante ao exposto em [ABNT 2008] e [Alberts and Audrey 2002], e considera apenas a probabilidade de impacto negativo, assim pode-se determinar risco de segurança da informação como a combinação da probabilidade de uma determinada ameaça explorar uma vulnerabilidade de um ativo (evento) com o impacto de suas potenciais consequências.

No processo de gestão de riscos de segurança da informação, conforme proposto em [ABNT 2008], o risco de segurança da informação é concebido por meio da definição de cenários de incidentes de segurança da informação, que são descrições fictícias de um potencial conjunto de incidentes que uma organização pode estar sujeita. A este cenário são associados os ativos, as vulnerabilidades inerentes a esses, as ameaças que podem explorar essas últimas, os controles (existentes e potenciais), assim como as consequências, probabilidade de ocorrência e medida de impacto.

A preservação das propriedades e dos aspectos da informação anteriormente mencionados depende do estabelecimento de uma ação gerencial explícita, que é chamada de gestão da segurança da informação (GSI).

A GSI tem como objetivo principal fazer com que as ações e decisões relativas à segurança da informação estejam alinhadas aos objetivos e estratégias do negócio da organização e que estas sejam promovidas por meio de um conjunto de controles (tais como procedimentos, estruturas organizacionais, políticas etc) com objetivos específicos. Um Sistema de Gestão de Segurança da Informação (SGSI) visa justamente permitir que a organização que o implementa alcance seus objetivos relativos à segurança da informação. A própria norma ABNT NBR ISO/IEC 27001, [ABNT 2006] versa que um Sistema de Gestão de Segurança da Informação baseia-se numa análise de riscos para estabelecer, programar, operar, monitorizar, rever, manter e melhorar a Segurança da Informação.

Um processo de Gestão de Riscos de Segurança da Informação (GRSI) pode ser aplicado nas mais variadas esferas de uma organização, seja nela como um todo, em uma de suas divisões, ou apenas em um projeto específico. Em qualquer situação, esse ambiente onde a mesma será desenvolvida deve ser bem definido, de modo a permitir as de- 
cisões específicas e corretas que impliquem em ações eficientes nestes processos. Ou seja, o escopo de aplicação da gestão de riscos deve ser transparente e delineado. A definição de onde ou em que será implantada a gerência de riscos de segurança da informação é um dos itens mais importantes no que tange ao princípio de estabelecer uma base para um processo de gestão contínuo, conforme definido em [Alberts and Audrey 2002].

Em [ABNT 2008] é indicado um processo de GRSI, cujas principais atividades são: definição de contexto, apreciação de riscos (que prevê subatividades de identificação, estimativa e avaliação de riscos), tratamento de riscos e aceitação de riscos, além de uma comunicação efetiva entre as partes envolvidas, e um monitoramento e revisões contínuas ao longo do processo. Cada uma destas atividades deve ter suas entradas, ações e resultados registrados, pois esses registros são parte dos requisitos para uma GSI efetiva e consequentemente para uma boa governança de TI e corporativa.

\section{Ontologias}

Descrever e representar conceitos e propriedades relevantes em um domínio específico é uma das principais funcionalidades de uma ontologia. Por meio deste tipo de representação, facilita-se o compartilhamento de conhecimento em um domínio, visto estabelecer-se sobre um vocabulário, além de permitir aquisição de novos conhecimentos com base em axiomas e regras definidas para a ontologia proposta.

Segundo [Guarino 1998], uma ontologia pode ser entendida como um artefato de engenharia, baseado em vocabulário formal específico, cujo uso permite a descrição de um domínio que se quer representar. Consoante a esta definição, uma formalização do conceito de ontologia pertinente é a proposta por [Stumme and Maedche 2003] e complementada por [Ehrig et al. 2004], representada pela 10-upla abaixo:

$$
O:=\left(C, T, \leq_{C}, \leq_{T}, R, A, \sigma_{A}, \sigma_{R}, \leq_{R}, \leq_{A}\right)
$$

$\mathrm{Na}$ equação 1 acima, $\mathrm{C}$ refere-se ao conjunto de conceitos, $\mathrm{R}$ ao conjunto de relacionamentos e $\mathrm{T}$ ao conjunto de tipos de dados, estes três conjuntos são disjuntos. O conceitos estão organizados segundo uma hierarquia $\leq_{C}$, as relações segundo $\leq_{R}$ e os atributos segundo $\leq_{T}$. Os relacionamentos têm assinatura $\sigma_{R}: R \rightarrow \mathrm{C}$ x C. A é o conjunto dos atributos de tipos de dados associados a conceitos, com assinatura $\sigma_{A}$ : $A \rightarrow \mathrm{C} \mathrm{x}$ T e organizados segundo a taxonomia $\leq_{A}$.

Assim, com base na explicação da equação 1, explicitam-se alguns entendimentos: o conjunto de conceitos são representações de objetos (entidades) do mundo real, que possuem propriedades com outros objetos (propriedades relacionais ou relacionamentos) e propriedades descritivas (atributos com valores de determinados tipos de dados) que os descrevem (representando estados, eventos ou processos destas entidades). As instâncias de um conceito representam um objeto particular e a sua descrição (atributos e relacionamentos) dentro de um conjunto de objetos do mesmo tipo. O conjunto de instânciações associado a ontologia constitue uma base de conhecimento relativa aquele domínio.

Em [Fensel 2000], aponta-se que a definição de ontologia dada em [Gruber 1995] melhor representa a essência de uma ontologia como sistema de organização do conhecimento, que privilegia a conexão de conceitos e a representação dos relacionamentos complexos entre eles, conforme conceitua [Brascher and Carlan 2010]. Define-se em 
[Gruber 1995], que ontologia diz respeito a uma "especificação formal e explícita de uma conceitualização compartilhada". Conceitualização é concebida aqui como um modelo abstrato que representa um determinado contexto no mundo, por meio dos conceitos mais relevantes a este domínio. Enquanto compartilhada caracteriza esta conceitualização com um conhecimento consensual, comum ao grupo envolvido no domínio especificado. Ao passo que explícita refere-se à característica de os objetos representados pelo conjunto de conceitos, assim como os relacionamentos e atributos a eles associados, estarem definidos de forma explícita. E formal ao fato de a especificação ser declarativamente definida (por meio de lógica descritiva por exemplo) e assim ser compreensível a agentes e sistemas.

Podem-se citar como principais benefícios da utilização de ontologias, tanto no que tange a domínios relacionados à segurança da informação, como apontado em [Donner 2003], [R Andersson and Hallberg 2003], e [Raskin et al. 2001], quanto na sua utilização de uma forma geral, segundo [Noy and McGuinners 2001], os seguintes:

- Formalização de um fenômeno, por meio da organização e sistematização de conceitos relacionados ao domínio em questão, seus relacionamentos e atributos, propiciando um compartilhamento do entendimento comum a pessoas e agentes de software;

- Uma compreensão mais clara do domínio modelado, devido à definição formal e explícita, além da aquisição de conhecimento (geração automática) possibilitada por meio das regras de inferência e axiomas;

- A manutenção do conhecimento no modelo obtido (seja pela reuso ou extensão) também é facilitada pela especificação formal e explícita empregada nas ontologias;

- O compartilhamento de conhecimento e informações também é favorecido devido ao domínio alvo ser modelado com base em uma conceitualização compartilhada e permitir a instanciação dos conceitos e propriedades especificados;

- A utilização de ontologias permite separar conhecimento de um domínio do conhecimento operacional, assim uma mesma ontologia pode ser utilizada em variadas aplicações, além de admitir a interoperabilidade entre elas.

\section{O processo de desenvolvimento da InfoSecRM}

Para a elaboração da InfoSecRM foram utilizadas as perspectivas de três abordagens, a metodologia methontology [Fernandez-Lopez et al. 1997] como o processo que define o arcabouço das atividades a serem realizadas, o método 101 [Noy and McGuinners 2001] para definir o "como fazer" de algumas destas atividades (na conceituação por exemplo, onde os passos são bem detalhados) e a metodologia proposta por Fox e Gruninger no projeto TOVE [Grüninger and Fox 1995], com a utilização das idéias de cenários de motivação e de questões de competência por ela proposta.

Para a implementação da InfoSecRM foram utilizadas as linguagem OWL-DL e SPARQL, o framework Protégé 3.4.7 e a máquina de inferência Pellet 1.5.2.

A InfoSecRM teve como idéia base o conceito de risco associado a um cenário de incidente de segurança da informação. Assim, um risco está associado a um cenário de incidente de segurança da informação, que é uma descrição fictícia de um potencial 
conjunto de incidentes que uma organização pode estar sujeita. A este cenário são associados os ativos, as vulnerabilidades inerentes a esses, as ameaças que podem explorar essas últimas, os controles, assim como as consequências, probabilidade de ocorrência e medida de impacto. Com base nestes é estimado o nível de risco. A representação do núcleo da InfoSecRM descrito pode ser observada na figura 1.

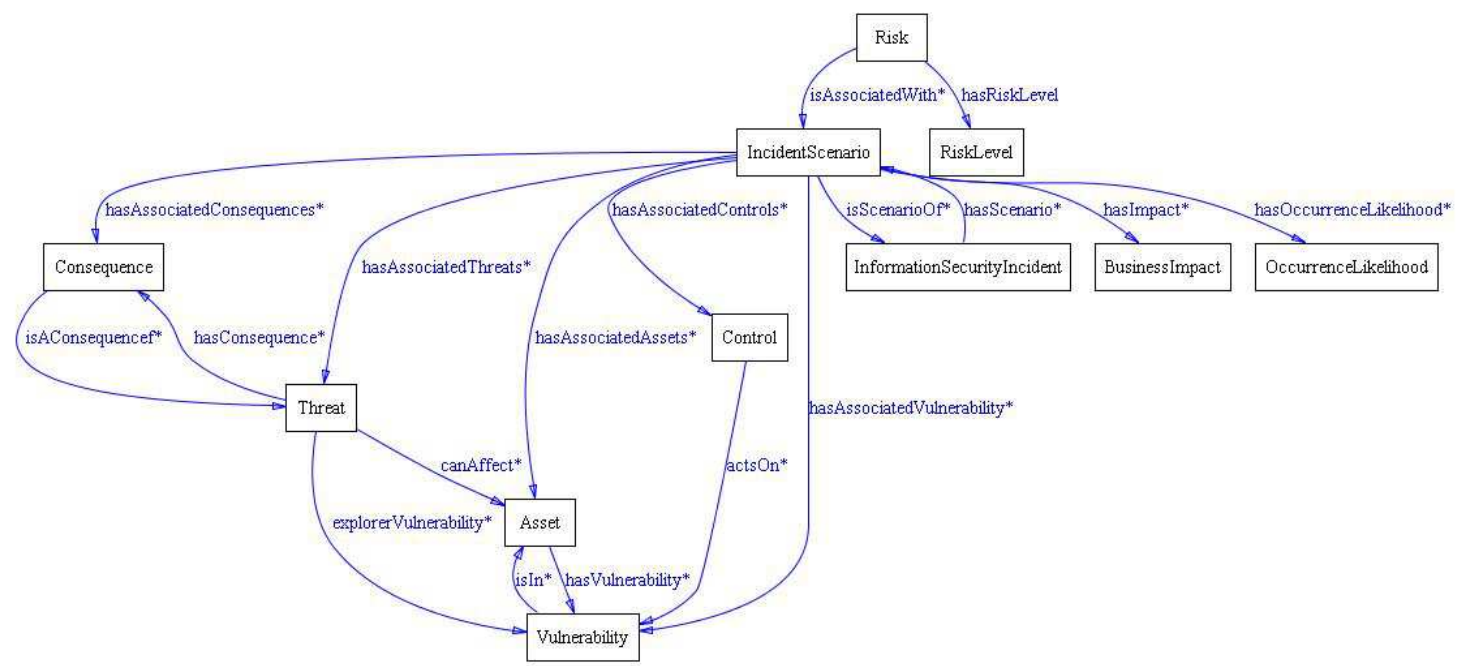

Figure 1. Representação do núcleo de conceitos e relacionamentos que formam a base da InfoSecRM

Além da idéia central, outra elemento importante na concepção da InfoSecREM, foi o processo de gestão de riscos de segurança da informação definido na norma ISO 27005 [ABNT 2008].

Com base nas duas idéias citadas, desenvolveu-se a InfoSecRM. Foram elencados os termos mais importantes a serem representados pela ontologia, definidas classes e suas hierarquias, e as propriedades de cada classe e suas restrições. A InfoSecRM possui 88 classes (82 classes nomeadas e 6 definidas), 68 relacionamentos e 9 atributos.

Exemplo de classe desta ontologia:

Classe Risk - Representam os riscos (foco do processo de gestão de riscos) associados a indivíduos da classe "IncidentScenario". Possui as seguintes propriedades:

- isARiskAssociatedWith - identifica o cenário de incidente de segurança da informação associado ao risco.

- hasRiskLevel - identifica o nível de risco de um indivíduo da classe Risk, atribuído quando da atividade de estimativa de risco (pode ser alto, médio ou baixo).

- hasPriority - identifica a prioridade dada a um risco, na atividade de avaliação de risco, para que este seja tratado (pode ser uma prioridade alta, média ou baixa).

- hasTreatment - indica o tratamento dado a um risco quando da atividade de tratamento de risco.

- hasRiskAcceptanceStatus - representa se um risco foi ou não aceito durante a atividade de aceitação de risco.

- hasResponsible - associa a responsabilidade do risco a um colaborador ou fornecedor. 
- hasDescription - apresenta uma string de descrição do risco.

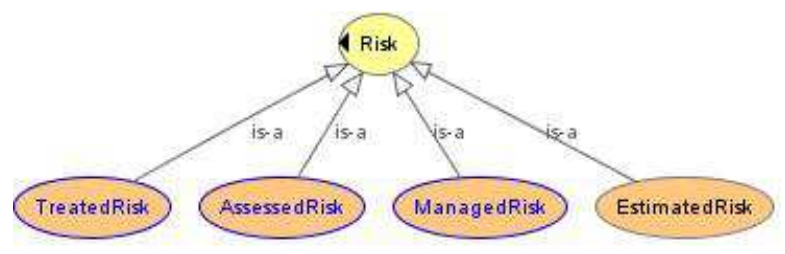

Figure 2. Hierarquia da classe Risk

A classe Risk (classe nomeada) possui a hierarquia representada na figura 2. As suas subclasses (classes definidas) identificam quais atividades já foram realizadas sobre um determinado risco. Por exemplo: a classe TreatedRisk possui as condições necessárias e suficientes, indicadas na equação 2 :

TreatedRisk $\equiv$ Risk $\cap \forall$ isAssociatedWith.IncidentScenario $\cap \exists$ hasLevel.RiskLevel $\cap$

$\exists$ hasPriority.RiskPriority $\cap \exists$ hasTreatment.Treatment

Denota-se, das condições expressas acima, que os riscos inferidos como indivíduos da classe TreatedRisk já passaram pela atividade de tratamento de risco.

\section{Avaliação da InfoSecRM e análise dos benefícios de sua utilização}

O processo de avaliação de ontologias consiste em atividades de verificação e de validação, que, segundo define [Obrst et al. 2007], referem-se respectivamente a: avaliar se a ontologia implementa os requisitos corretamente (avaliar quanto à forma) e avaliar se a ontologia modela de fato o domínio alvo (avaliar se a ontologia correta foi construída).

As atividades de verificação foram guiadas pela abordagem indicada em [Lozano-Tello and Gomez-Perez 2004] e em [Sirin et al. 2007], onde é proposto avaliar ontologias segundo um conjunto de critérios pré-definidos, e também pela abordagem proposta em [Maedche et al. 2002] e discutida em [Obrst et al. 2007], que indica a comparação entre ontologias de um domínio relacionado como forma de verificação (com base em uma análise quantitativa).

Considerando o exposto por [Vrandeĉić 2009], onde é afirmado que a validação requer uma colaboração entre os profissionais responsáveis pelo desenvolvimento da ontologia e os profissionais do domínio mapeado, para as atividades de validação, adotou-se a abordagem proposta em [Obrst et al. 2007], que avalia a capacidade da ontologia criada de responder a algumas das questões de competência. Nesse sentido, a ontologia foi utilizada nas atividades de um processo de GRSI em uma gerencia operacional do departamento de TI de uma organização governamental, com vistas a instanciar suas classes e relacionamentos, e assim, permitir as pesquisas pelas questões de competência. Esta abordagem é similar à adotada em [Silva et al. 2011], porém foi realizada com dados reais de um processo de GRSI de uma organização

Para a validação, ainda foi adotada a abordagem denominada Data-driven, proposta em [Brewster et al. 2004], que consite em comparar a ontologia desenvolvida com um conjunto de dados ou documentos sobre o domínio modelado. 


\subsection{Verificação}

A ontologia foi verificada quanto aos critérios: acurácia, adaptabilidade, transparência, granularidade, adequação organizacional, classificação, consistência, expressividade, precisão, satisfação, usabilidade e utilidade, propostos em [Vrandeĉić 2009] e [Lozano-Tello and Gomez-Perez 2004].

Para a verificação do critério expressividade, por exemplo, observou-se que a InfoSecRM possui interseção e disjunção entre classes, quantificação universal e existencial, negação, regras transitivas, inversas e funcionais, hierarquia de classes e de propriedades, definição de classes por enumeração, restrições de cardinalidade e utilização de tipos de dados. Em outras palavras, identificou-se que ontologia verificada possui expressividade SHOIN(D) que é a maior expressividade que a OWL-DL pode proporcionar.

Já com relação à verificação por comparação entre ontologias, considerando a abordagem, proposta por [Maedche et al. 2002], de comparar a InfoSecRM com outras ontologias, adotou-se como métrica a indicada em [Ning and Shihan 2006], que é realizada com base nos indicadores: Quantidade de classes nomeadas, Média de propriedades do tipo objeto (relacionamentos), Nível da ontologia quanto a hierarquias - relacionamento is-a (é um) e Classe com maior número de relacionamentos $i s-a$ da ontologia.

Foram utilizadas para este comparativo as ontologias propostas em [Martimiano 2006] e [Azevedo 1994], respectivamente, OntoSec e CoreSec. A OntoSec representa parte do domínio de segurança da informação, porém seu foco é na gestão de incidentes de segurança da informação. Já a CoreSec tem como domínio a segurança da informação, propõe uma representação com conceitos de alto nível, facilitando a sua utilização em avaliação de riscos e gestão de segurança da informação.

Por meio destes indicadores, pode-se observar que a estrututa da InfoSecRM assemelha-se mais à CoreSec do que à OntoSec, o número de classes nomeadas (respectivamente, 82,89 e 59) e os conceitos representados indicam esta semelhança. No entanto, é importante frizar que enquanto o domínio modelado pela InfoSecRM refere-se apenas à gestão de riscos de segurança da informação, o domínio modelado pela CoreSec tinha como objetivo abranger toda a gestão de segurança da informação.

Observou-se também que a InfoSecRM apresenta maior média de propriedades do tipo objeto por classes nomeadas (0.82, frente a 0.47 da OntoSec e 0.45 da CoreSec). Este número é um indicador de que a conceitualização proposta por esta ontologia representa bem as relações semânticas entre os conceitos do domínio representado, como pode ser observado nas propriedades que relacionam os conceitos ativo, vulnerabilidade, ameaças, consequência, incidente, cenário de incidente e riscos por exemplo.

Com relação à quantidade de níveis e à classe com maior número de relacionamentos is- $a$, verificou-se que ontologia proposta neste trabalho possui seis níveis em sua hierarquia, enquanto a OntoSec e a CoreSec possuem 5. Neste sexto nível estão representadas as subclasses das classes Datamedium, Network e Host, que são subclasses da classe Hardware, que é uma subclasse da classe PhysicalAsset, que por sua vez é uma subclasse da classe Asset. A classe Asset é a classe na InfoSecRM que possui mais subclasses, ou seja, mais relacionamentos do tipo is- $a$. Este grau de especialização desta classe justifica-se em parte devido ao fato de o inventário de ativos e a sua valoração para uma organização serem atividades críticas ao processo de GRSI. 
Uma constatação importante de ser citada é que na OntoSec e na CoreSec a maioria dos relacionamentos se concentra na classe que representa os incidentes de segurança (Securityincident). Na InfoSecRM, esta tendência de centralização em incidentes de segurança da informação também é perceptível, no entanto a dinâmica empregada considera estes incidentes como pertencentes a cenários de incidentes e estes por sua vez como associados a riscos, daí a maioria dos relacionamentos da ontologia descrita neste trabalho concentrar-se nas classes Risk e IncidentScenario.

\subsection{Validação}

A InfoSecRM foi utilizada nas atividades de um processo, ainda de nível inicial, de GRSI em uma gerencial operacional do departamento de TI de uma organização governamental. Esta organização ainda não possui política de segurança da informação ou outros documentos que possam balizar as ações destes processo em todas as suas áreas e com a participação de todos os colaboradores. Assim o processo limitou-se à área que gerencia a infraestrutura de TI e às questões onde os colaboradores participantes tinham autonomia e autoridade para fazê-lo.

Os conceitos e relacionamentos representados na InfoSecRM foram instanciados representando as ações do processo de GRSI com as limitações descritas acima. Por meio de consultas SPARQL às instanciações realizadas, pode-se responder às questões de competência definidas durante o desenvolvimento desta ontologia.

Exemplo de questão de competência:

Quais são os riscos de nível alto a que uma organização está sujeita?

SELECT ?Risk

WHERE \{?Risk:hasRiskLevel:High_Risk\}

As respostas a questões de competência, como a citada, permitem, não apenas verificar que os conceitos necessários à representação do domínio de GRSI foram modelados pela ontologia, como também auxiliar os usuários da InfoSecRM a buscar informações nas bases de conhecimento geradas sobre a estrutura que ela representa. Assim foi possível observar que a utilização da InfoSecRm permitiu auxiliar na implementação da GRSI, ao armazenar as informações e indicar as atividades e escopo deste processo, apresentando-se como uma estrutura padronizada por meio da qual pode-se operar sobre os conceitos de segurança da informação relacionados à gestão de riscos e permitir o compartilhamento das informações relacionadas.

A ontologia desenvolvida ainda auxiliou no aprendizado, de alguns colaboradores, dos conceitos envolvidos em um processo de gestão de riscos de segurança da informação. A ontologia permite uma representação intuitiva dos conceitos do domínio de GRSI, suas respectivas propriedades e atriutos, além de incluir pequenas descrições para cada um destes elementos representados. Este tipo de conceitualização, juntamente com os plugins de visualização utilizados, tornam a percepção do domínio modelado mais clara.

Durante as atividades de validação, a InfoSecRM foi ainda comparada com documentos sobre o domínio modelado. Para esta abordagem de validação foram utilizadas as normas ABNT 27002 [ABNT 2005], ABNT 27001 [ABNT 2006] e ABNT 27005 [ABNT 2008] 
Estas normas foram utilizadas como os documentos sobre o domínio representado por se tratarem de integrantes da família de normas ISO 27000 que destina-se a auxiliar organizações a implementar e operar um sistema de gerenciamento de segurança da informação (SGSI). Como já exposto, um processo de GRSI é um fator crítico a um SGSI, pois por meio do mesmo pode-se implementar e monitorar os controles de segurança da informação de uma organização, além de promover ações que os melhorem continuamente.

Por meio da comparação da InfoSecRM às normas citadas, pode-se notar que os aspectos mais relevantes do contexto de gerenciamento de riscos de segurança da informação foram representados, principalmente no que tange ao conceito de riscos e cenários de incidentes de segurança da informação, e ao processo de GRSI em si.

\section{Conclusões e Trabalhos Futuros}

A InfoSecRM, ontologia de domínio desenvolvida neste trabalho, apresenta uma conceitualização do conhecimento relacionado à GRSI. Por meio desta podem ser instanciados os conceitos envolvidos (como riscos, cenários de incidentes, impacto etc) e também as atividades propostas para um processo de gestão de riscos (como análise de riscos, avaliação de riscos, tratamento de riscos etc). As representações desta ontologia auxiliam não só a tomada de decisões neste domínio, como a própria implementação e continuidade deste processo. Assim, as principais contribuições deste trabalho foram:

- Uma conceitualização formal, desenvolvida e avaliada segundo um processo bem definido, que apresenta uma representação das informações relacionadas à gestão de riscos de segurança da informação. Por meio desta representação, promove-se a aquisição e o compartilhamento de informações e conhecimento neste domínio.

- Promoção do processo de GRSI em organizações por meio da utilização da InfoSecRM, que como visto pode contribuir na implementação de uma gestão de riscos e na tomada de decisões, e ser utilizada na criação e estruturação de uma base de conhecimento de riscos de segurança da informação.

- Reúso de Conhecimento e informações, visto que a ontologia desenvolvida pode ser utilizada em processos de GRSI de organizações, em treinamentos de colaboradores em GRSI, para o desenvolvimento de novas ontologias (de aplicação por exemplo) e como base para aplicações.

Diante das contribuições e resultados obtidos, indentificaram-se as seguintes oportunidades de trabalhos futuros:

- Desenvolver um sistema que permita auxiliar em processos de GRSI, indicando os conceitos trabalhados e as informações necessárias nos moldes do realizado neste trabalho, tendo como base a InfoSecRM. Por meio deste, fornecer uma interface ainda mais intuituiva e com uma estrutura mais robusta para usuários menos familiarizados com conceitos de segurança da informação e com a utilização de ontologias.

- Expandir os conceitos da InfoSecRM de forma a representar também o indicado nas outras normas da família ISO 27000.

- Desenvolver ontologias de aplicação, apartir da InfoSecRM, para cenários mais específicos relacionados à GRSI, como por exemplo as atividades de inventário de ativos. 


\section{References}

ABNT (2005). ABNT NBR ISO/IEC 27002 - Código de prática para a gestão de segurança da informação. Associação Brasileira de Normas Técnicas, Rio de Janeiro.

ABNT (2006). ABNT NBR ISO/IEC 27001 - Sistemas de gestão de segurança da informação - requisitos. Associação Brasileira de Normas Técnicas, Rio de Janeiro.

ABNT (2008). ABNT NBR ISO/IEC 27005 - Gestão de riscos de segurança da informação. Associação Brasileira de Normas Técnicas, Rio de Janeiro.

Alberts, C. and Audrey, D. (2002). Managing Information Security Risks: The OCTAVE Approach. Addison Wesley.

AS/NZS (2004). AS/NZS 4360:2004 - Risk Management. Australian/New Zealand Standard, Autralia: GPO Box 5420, Sydney / New Zealand: Private Bag 2439, Wellington 6020 .

Azevedo, R. R. (1994). Coresec: Uma ontologia para o domínio de segurança da informação. Master's thesis, Universidade Federal de Pernambuco, Recife.

Brascher, M. and Carlan, E. (2010). Passeios no Bosque da Informação: Estudos sobre Representação e Organização da Informação e do Conhecimento, chapter Sistemas de organização do conhecimento: antigas e novas linguagens, pages 147-176. IBICT, Brasília.

Brewster, C., Alani, H., Dasmahapatra, S., and Wilks, Y. (2004). Data-driven ontology evaluation. In Proceedings of the Language Resources and Evaluation Conference (LREC 2004), pages 164-168, Lisbon, Portugal. European Language Resources Association.

Donner, M. (2003). Toward a security ontology. IEEE Security and Privacy magazine, $1(3): 6-7$.

Ehrig, M., Haase, P., Hefke, M., and Stojanovic, N. (2004). Similarity for ontologies - a comprehensive framework. FZI Research Center for Information Technologies at the University of Karlsruhe.

Fensel, D. (2000). Ontologies: Silver Bullet for Knowledge Management and Eletronic Commerce. Springer.

Fernandez-Lopez, M., Gomez-Perez, A., and Juristo, N. (1997). Methontology: from ontological art towards ontological engineering. In Proceedings of the AAAI97 Spring Symposium, pages 33-40, Stanford, USA.

Gruber, T. R. (1995). Toward principles for the design of ontologies used for knowledge sharing. International Journal of Human-Computer Studies, 43(5/6):907-928.

Grüninger, M. and Fox, M. (1995). Methodology for the Design and Evaluation of Ontologies. In IJCAI'95, Workshop on Basic Ontological Issues in Knowledge Sharing, April 13, 1995.

GSI-PR (2008). Gabinete de Segurança Institucional da Presidência da República. Norma Complementar GSI $n^{\circ}$ 2, de 14 de outubro de 2008. Gabinete de Segurança Institucional da Presidência da República. 
Guarino, N. (1998). Formal ontologies and information systems. First International Conference (FOIS), 1:3-15.

ISO (2009). ISO/IEC 27000 - Information Security Management Systems. International Organization for Standardization.

Lozano-Tello and Gomez-Perez, A. (2004). ONTOMETRIC: A method to choose the appropriate ontology. JOURNAL OF DATABASE MANAGEMENT, 15(2):1-18.

Maedche, A., Maedche, E., and Staab, S. (2002). Measuring similarity between ontologies. In in Proceedings of the European Conference on Knowledge Acquisition and Management (EKAW, pages 251-263. Springer.

Martimiano, L. A. F. (2006). Sobre a estruturação de informação de segurança computacional: o uso de ontologia. PhD thesis, Instituto de Ciências Matemáticas e de Computação - ICMC, Universidade de São Paulo - USP, São Carlos.

Ning, H. and Shihan, D. (2006). Structure-based ontology evaluation. In e-Business Engineering, 2006. ICEBE '06. IEEE International Conference on, pages 132-137.

Noy, N. F. and McGuinners, D. L. (2001). Ontology development 101: A guide to create your first ontology. Knowledge Systems Laboratory - Stanfor University.

Obrst, L., Ceusters, W., Mani, I., Ray, S., and Smith, B. (2007). The evaluation of ontologies. In Baker, C. J. and Cheung, K.-H., editors, Revolutionizing Knowledge Discovery in the Life Sciences, chapter 7, pages 139-158. Springer.

R Andersson, A. H. and Hallberg, J. (2003). Evaluation of the Security of Components in Distributed Information Systems. Swedish Defence Reaserch Agency.

Raskin, V., Hempelmann, C. F., Triezenberg, K. E., and Nirenburg, S. (2001). Ontology in information security: a useful theoretical foundation and methology tool. Proceedings of the workshop and New Security Paradigms.

Schumacher, M. (2003). Secuurity Engineering with patterns - Origins, Theoretical and New Applications, chapter Toward security core ontology, pages 87-96. Simpreger Verlag.

Silva, P. F., Otte, H., Todesco, J. L., and Gauthier, F. A. O. (2011). Uma ontologia para gestão de segurança da informação. Ontobras/Most - Internacional Workshop on Metamodels Ontologies and Semantic Technologies.

Sirin, E., Parsia, B., Grau, B., Kalyanpur, A., and Katz, Y. (2007). Pellet: A practical OWL-DL reasoner. Web Semantics: Science, Services and Agents on the World Wide Web, 5(2):51-53.

Sêmola, M. (2003). Gestão da segurança da informação: visão executiva da segurança da informação. Elsevier, Rio de Janeiro.

Stumme, G. and Maedche, A. (2003). Fca-merge: Bottom-up merging of ontologies. 17th Intl. Conf. on Artificial Inelligence (IJCAI '01, 19:225-230.

Vrandeĉić, D. (2009). Ontology Evaluation, pages 293-313. International Handbook on Information Systems. Springer. 